OPEN ACCESS

Edited by:

Patricia Maria Lourenço Dutra,

Rio de Janeiro State University, Brazil

Reviewed by:

Wagner Luiz Prado,

California State University,

San Bernardino, United States Kate Edwards,

The University of Sydney, Australia

${ }^{*}$ Correspondence: Emily C. LaVoy eclavoy@uh.edu

Specialty section:

This article was submitted to

Exercise Physiology,

a section of the journal

Frontiers in Physiology

Received: 22 May 2021

Accepted: 21 July 2021

Published: 12 August 2021

Citation:

Elzayat MT, Markofski MM,

Simpson RJ, Laughlin M and LaVoy EC (2021) No Effect of Acute Eccentric Resistance Exercise on Immune Responses to Influenza Vaccination in Older Adults:

A Randomized Control Trial.

Front. Physiol. 12:713183. doi: 10.3389/fphys.2021.713183

\section{No Effect of Acute Eccentric Resistance Exercise on Immune Responses to Influenza Vaccination in Older Adults: A Randomized Control Trial}

\author{
Mahmoud T. Elzayat' ${ }^{1}$, Melissa M. Markofski ${ }^{1}$, Richard J. Simpson 1,2, Mitzi Laughlin ${ }^{1,3}$ \\ and Emily C. LaVoy ${ }^{1 *}$ \\ ' Department of Health and Human Performance, University of Houston, Houston, TX, United States, ${ }^{2}$ Department \\ of Nutritional Sciences, College of Agriculture and Life Sciences, University of Arizona, Tucson, AZ, United States, ${ }^{3}$ Fondren \\ Orthopedic Research Institute, Houston, TX, United States
}

Introduction: Older adults are at elevated risk for morbidity and mortality caused by influenza. Vaccination is the primary means of prophylaxis, but protection is often compromised in older adults. As resistance exercise mobilizes immune cells into muscle, it may enhance vaccination response.

Purpose: Compare antibody and cell mediated immune responses to influenza vaccination in older adults who performed eccentric resistance exercise immediately prior to vaccination to those who did not exercise.

Methods: Twenty nine resistance training-naive older adults (20 women, $73.9 \pm 5.3$ years) were randomized to 1 of 3 groups: vaccination in the same arm that exercised (Ex-S), vaccination in the opposite arm that exercised (Ex-Op), and seated rest (NoEx). Exercise consisted of 10 sets of 5 eccentric unilateral repetitions at $80 \%$ of the pre-determined concentric one repetition maximum. Lateral raises were alternated with bicep curls. No-Ex sat quietly for 25 min. Following exercise or rest, all received the 2018 quadrivalent influenza vaccine (Seqirus Afluria) in the non-dominant deltoid. Antibody titers against each influenza vaccine strain were determined by hemagglutinin inhibition assays at baseline, 6-, and 24-weeks post-vaccination. Influenza-specific T cells were quantified after stimulation with the vaccine by intracellular cytokine staining.

Results: No significant group $x$ time effects were found in antibody responses to any strain (interaction for $\mathrm{A} / \mathrm{H} 1 \mathrm{~N} 1: p=0.682$; $\mathrm{A} / \mathrm{H} 3 \mathrm{~N} 2: p=0.644$; B/Colorado/06/2017: $p=0.262 ; \mathrm{B} /$ Phuket/3073/2013: $p=0.851)$. Groups did not differ in fold-increase of antibody titers 6- and 24-weeks post-vaccination. Influenza-specific T-cells did not differ between groups at any time (comparison at baseline: $p=0.985$; 6-weeks: $p=0.889 ; 24$ weeks: $p=0.857$ ). One subject (Ex-S) reported flu-like symptoms 18 weeks post-vaccination. 
Conclusion: Acute arm eccentric exercise did not influence antibody titers or cell mediated immune responses to the influenza vaccine delivered post-exercise in older adults. More strenuous exercise may be required for exercise to act as an adjuvant.

ClinicalTrials.gov Identifier: NCT03736759.

Keywords: immunity, virus, exercise, aging, vaccine

\section{INTRODUCTION}

The aging immune system leaves older adults more vulnerable to infections, including with influenza virus. Influenza infection is associated with the death of thousands of people in the United States each year, approximately $90 \%$ of which occur among older adults ( $\geq 65$ years) (Thompson et al., 2003, 2004, 2009). The primary means of protection against influenza is through annual vaccination with the seasonal influenza vaccine. Unfortunately, protective responses are impaired in this population, which likely accounts for the increased prevalence of infection in older adults (Goodwin et al., 2006; Song et al., 2010; Castilla et al., 2013). One common approach to assess protection is measurement of influenza-specific antibody titers. Older adults exhibit lower antibody responses to the influenza vaccine leading to a clinical vaccine efficacy of just $17-53 \%$, compared to $70-90 \%$ efficacy in younger adults (Goodwin et al., 2006; Castilla et al., 2013). Furthermore, antibody titers to influenza may decline more rapidly in older adults, meaning the effectiveness of the influenza vaccine may be further reduced if influenza exposure occurs late in the season (Song et al., 2010). Cell mediated immunity provided by influenza-specific cytotoxic T-cells also offers protection against disease, maybe induced by vaccination (Ennis et al., 1981; Sridhar et al., 2013). Unfortunately, impaired T-cell responses after influenza vaccination have been reported in older adults relative to younger adults (Fagiolo et al., 1993). This reduction in vaccination response is present even in older adults without chronic disease (Fagiolo et al., 1993; Hainz et al., 2005; Van Epps et al., 2017).

Considering the importance of generating protective immune responses against influenza infection, the development of strategies to improve vaccine responses among older adults is vital. Deficits in the antibody response to vaccines arise from agerelated declines in the function of B-cells and T-cells (Haynes and Swain, 2006; Siegrist and Aspinall, 2009), and potentially, antigen-presenting cells including dendritic cells (Shurin et al., 2007). Different formulations of the influenza vaccine for older adults have been proposed to enhance the protective levels of immune responses, such as the use of different adjuvants, different modes of administration, and/or increased antigen dose in the vaccine (Banzhoff et al., 2003; Keitel et al., 2006; LerouxRoels et al., 2007; Della Cioppa et al., 2014). Although these strategies are typically more immunogenic than conventional non-adjuvanted influenza vaccines, they are also associated with greater injection site symptoms (e.g., erythema, swelling, and pain) and general systemic symptoms (e.g., malaise) postvaccination (Banzhoff et al., 2003; Keitel et al., 2006; Leroux-Roels et al., 2007; Della Cioppa et al., 2014). While the safety profile is clinically acceptable, increases in the potential discomfort of vaccination could reduce vaccine uptake. Further, increases in the antigen dose in certain vaccine formulations require increased manufacturing capabilities and thus may limit available vaccine doses. Thus, there is a need to develop a simple, cost-effective approach with minimal side-effects to enhance influenza vaccine responses in older adults.

One potential method to enhance vaccine responses in older adults may be through localized resistance exercise designed to induce mild transient muscle damage. Eccentric exercise, the application of tension as a muscle lengthens, reliably causes mild muscle damage and a local inflammatory response consisting of increased blood flow, vascular permeability, and immune cell invasion into the targeted muscle (Proske and Morgan, 2001; Peake et al., 2005). The inflammatory response is especially noted in resistance-training naïve individuals (Nosaka et al., 2001; Peake et al., 2005). In young adults, resistance exercise involving eccentric contractions of the deltoid and biceps bracchi muscles immediately prior to inoculation at this site improves antibody titer responses to vaccination (Edwards et al., 2007, 2010, 2012). While a recent study in older adults failed to find an effect of moderate-intensity whole-body resistance training on seroprotection, it may be that this exercise protocol was insufficient at eliciting an immune response (Bohn-Goldbaum et al., 2020). A strategy employing acute eccentric resistance exercise targeting the inoculation site has yet to be implemented in older adults who are more likely to benefit from these adjuvant effects of exercise. Moreover, it has not been determined from these published studies if the exercise effects are due to a local or a systemic response, as only the exercised arm has been inoculated. Eccentric resistance exercise also causes change in systemic immune parameters, such as increases in circulating leukocytes, altered expression of migration and adhesion molecules on neutrophils and monocytes, and increases in pro-inflammatory cytokines (Peake et al., 2005). Therefore, exercise could act as an adjuvant through generalized immune upregulation.

The aim of this study was to determine the impact of a single bout of unaccustomed eccentric arm/shoulder resistance exercises performed immediately prior to inoculation on antibody and cell mediated immune responses to the seasonal influenza vaccine in older adults. We also aimed to compare the effect of identical bouts of exercise prior to vaccination in the inoculated arm and the non-inoculated arm. We hypothesized that vaccination in the arm that performed the eccentric resistance exercise would increase antibody titers and influenzaspecific T-cells at 6 and 24 weeks post-vaccination relative to noexercise and vaccination in the arm that did not exercise. These experiments will allow us to determine if a single bout of eccentric resistance exercise can be used as a simple and inexpensive vaccine adjuvant, with low side-effects, in community dwelling 
older adults. These experiments will also help differentiate between the local and systemic effects of eccentric exercise on vaccine enhancement.

\section{MATERIALS AND METHODS}

\section{Study Design and Participants}

This parallel randomized controlled trial (ClinicalTrials.gov Identifier: NCT03736759) compared vaccine responses at three time points across three groups. Data were collected October 2018 through April 2019 in Houston, TX, United States. Men and women aged $\geq 65$ years were recruited from the metropolitan region of Houston, TX. Participants were screened to ensure they were non-frail (screened using Fried's criteria; Fried et al., 2001), non-smokers (>10 years), were not institutionalized, and met the American College of Sports Medicine criteria for participation in exercise (American College of Sports Medicine, et al., 2005). Participants were excluded if they reported: (i) a history of immune disease or vaccine-related allergies, (ii) having a physician-confirmed influenza infection in the prior year, (iii) regular use of medications known to affect the immune system, (iv) were bedridden in the prior 3 months, (v) engagement in resistance arm exercises in the prior 6 months, (vi) an impairment limiting exercise or prohibiting informed consent, and vii) already vaccinated with the 2018 seasonal influenza vaccine. This population was expected to manifest age-attenuated responses to vaccination but be able to safely complete the bout of eccentric exercise. All participants were naïve to resistance training of the upper body to preclude the repeated bout effect potentially minimizing inflammatory responses (Nosaka et al., 2001). This study was approved by the Institutional Review Board at the University of Houston (STUDY00000542) and was carried out in accordance with the Code of Ethics of the World Medical Association (Declaration of Helsinki).

Fifty-eight participants were initially screened for eligibility by phone or email; 16 did not meet inclusion criteria, and 12 declined to participate further or had schedule conflicts (Figure 1). Thirty participants were further screened in-person during the beginning of the first visit. Screening included a blood-pressure measurement, a survey of health behaviors and vaccine questions, the Mini-Mental State Examination, Fried's Frailty Criteria (including handgrip strength determination using a hydraulic hand dynamometer), and the ACSM/AHA Exercise Readiness Questionnaire. One participant was excluded from further participation due to exclusion criteria. The nature of the study and possible consequences of participation were discussed with each participant, and 29 participants provided informed consent. Participants were randomly assigned to one of three groups at a 1:1:1 ratio, stratified by sex: (Thompson et al., 2004) non-exercise inoculated control (NoEx); (Thompson et al., 2003) vaccination in the same arm that performed eccentric exercise of the deltoid and biceps bracchi (Ex-S); and (Thompson et al., 2009) vaccination in the opposite arm that performed eccentric exercise of the deltoid and biceps bracchi (Ex-Op). Randomization was achieved via a random number string generated in Microsoft Excel following the recommendations for adaptive randomization by Hoare et al. (2013). All participants completed Visit 1 in October 2018.

Participants completed two additional visits 6- and 24-weeks post-vaccination to provide a blood sample. These visits were expected to correspond to peak antibody-responses (6-weeks post-vaccination) (Rastogi et al., 1995) and also indicate if protection was provided for the entire influenza season (24weeks post-vaccination). Participants were compensated for their participation in each of the three visits with a gift-card.

\section{Intervention and Vaccination}

Following screening and consent process during Visit 1, participants completed questionnaires relating to life-stress and health behaviors (Perceived Stress Scale (Cohen et al., 1983), General Health Questionnaire-28 (Goldberg, 1978), RAND 36Item Health Survey (Hays et al., 1993), and a venous blood sample from the arm.

Participants assigned to Ex-S and Ex-Op completed single arm estimated 1 repetition maximum (1RM) tests for the bicep curl and lateral raise exercises. Briefly, participants completed 810 unassisted repetitions of the lateral raise exercise (shoulder abduction to $90^{\circ}$ with elbow extension) and bicep curl exercise (elbow flexion) using dumb bells of increasing weight until 8 repetitions could not be completed with proper technique. The 1RM for each exercise was estimated using the Brzycki Formula (Brzycki, 1993).

After the experimental resistance exercise load was determined, participants completed 10 sets of 5 repetitions of the eccentric component of each movement at $80 \%$ of their estimated 1RM by lowering the dumbbell in a controlled manner over the course of $4 \mathrm{~s}$. A study team member assisted with the concentric component of the exercise. Each experimental set consisted of one set of lateral raise exercise alternated with one set of bicep curl exercise. Weight was lowered if technique suffered to ensure that participants could complete the same number of repetitions. Participants rested $15 \mathrm{~s}$ after each set of lateral raise exercise (intra-set rest) and $30 \mathrm{~s}$ after each set of bicep curl exercise (inter-set rest). Participants in Ex-S completed the exercises in their non-dominant arm whereas Ex-Op completed the exercises in their dominant arm. Testing and exercise were completed in approximately $25 \mathrm{~min}$. No-Ex participants rested quietly for $25 \mathrm{~min}$.

Following exercise or seated rest, all participants were provided the quadrivalent influenza vaccine developed for the 2018-2019 influenza season (Afluria quadrivalent influenza vaccine; Seqirus; Lot 02544611A). The quadrivalent vaccine included four virus strains: A/Michigan/45/2015 (H1N1), A/Singapore/INFIMH-16-0019/2016 (H3N2), $\mathrm{B} /$ Colorado/06/2017, and $\mathrm{B} / \mathrm{Phuket} / 3073 / 2013$. Injections were given intramuscularly, using a 25 gauge $25 \mathrm{~mm}$ needle, directly into the deltoid of the non-dominant arm.

Participants were asked to report muscle soreness level using a visual analog scale before and after the injection, as well as each day for the next 7 days after injection via a scheduled phone call with a study team member (secondary outcome). Participants were contacted monthly for the remainder of 


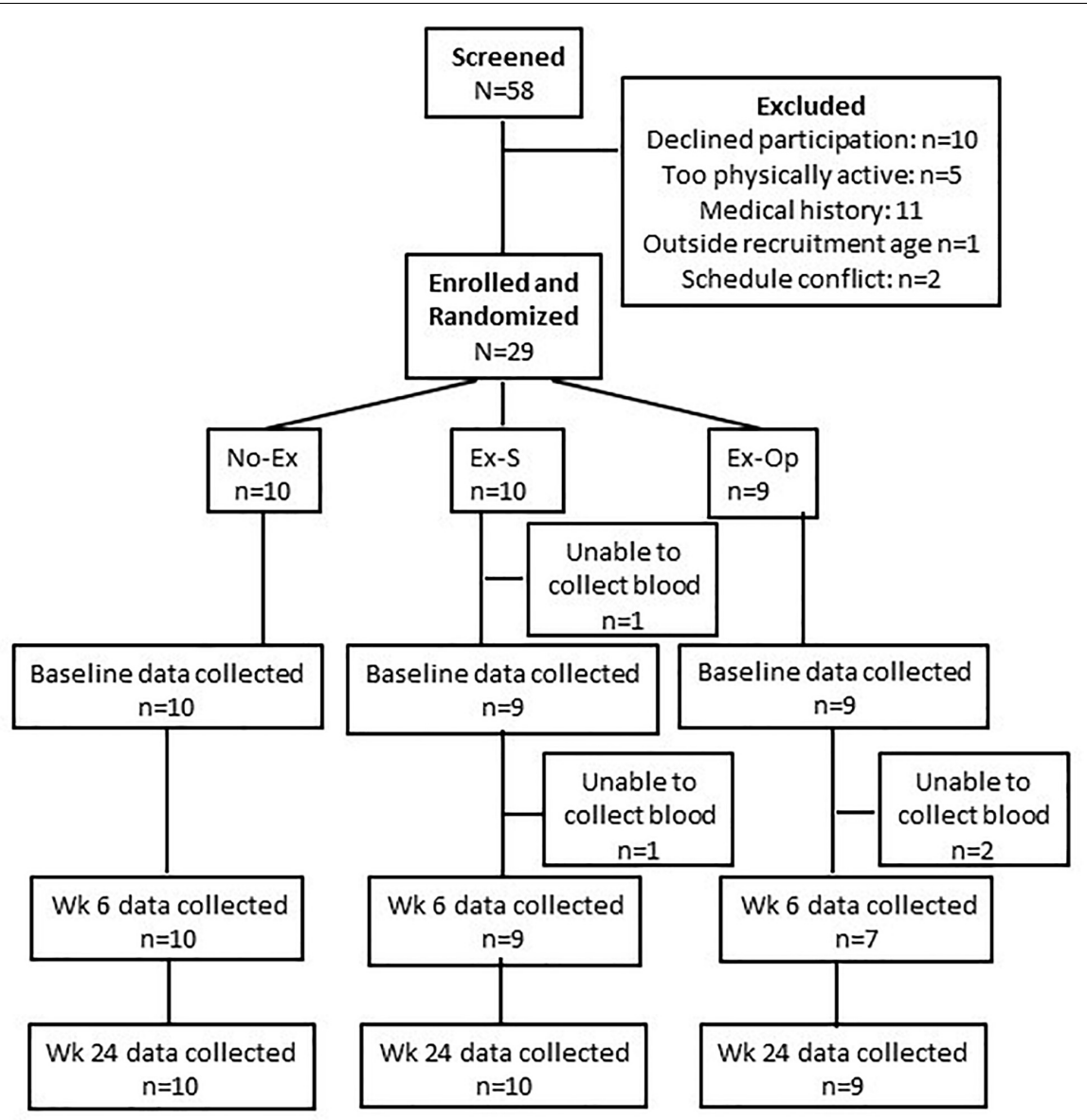

FIGURE 1 | CONSORT flow diagram.

the 6-month intervention to survey for flu-like symptoms (secondary outcome) and were asked to contact the study team if diagnosed with influenza.

\section{Hemagglutinin Inhibition Assays}

A primary outcome of this study was antibody titers to each influenza strain included in the vaccine. Venous blood was collected into $10 \mathrm{~mL}$ serum collection tubes (Vacutainer, BD) prior to vaccination (Baseline), 6 weeks-post vaccination, and 24 weeks-post vaccination. Serum was isolated by centrifugation from each sample within $2 \mathrm{~h}$ of blood draw, and stored at $-80^{\circ} \mathrm{C}$. Following the final blood collection, all samples were shipped overnight on dry ice to a commercial laboratory (Southern Research, Birmingham, AL) for the measurement of anti-influenza antibodies against the four influenza virus strains present in the vaccine. A standard microtiter hemagglutination inhibition assay was performed by researchers blinded to the intervention. Samples were analyzed in duplicate with a repeat performed as per standard operating procedure. Correctness of data was verified by an independent operator who did not perform the assay.

Fold-increase (post-vaccine titer/pre-vaccine titer), seroprotection (antibody titer $\geq 40$ ), and seroconversion
( $\geq 4$-fold increase) were calculated from the geometric mean antibody titers (Beyer et al., 2004).

\section{Cell Mediated Immunity Assays}

A second key outcome of this study was influenza-specific T-cells. Venous blood was collected into $10 \mathrm{~mL}$ collection tubes treated with Sodium Heparin (Vacutainer, BD) prior to vaccination, 6 weeks-post vaccination, and 24 weeks-post vaccination. Within $2 \mathrm{~h}$ of collection, peripheral blood mononuclear cells (PBMCs) were isolated using density gradient centrifugation (Histopaque1077, Sigma) and cryopreserved at $-80^{\circ} \mathrm{C}$ for later bulk analyses. On experiment day, PBMCs were thawed and incubated at a concentration of $1 \times 10^{6}$ cells $/ \mathrm{ml}$ in 5\%FBS-RPMI (Gibco) at $37^{\circ}$ with $5 \% \mathrm{CO}_{2}$. Cells were stimulated for $18 \mathrm{~h}$ with phorbol 12 myristate 13-acetate (PMA) and ionomycin, previously titrated vaccine, or RPMI (unstimulated). Monensin ( $2 \mu \mathrm{M}$; eBioscience) was added to all cells for the final $6 \mathrm{~h}$. After washing with PBS, cells were labeled with previously titrated fluorescently tagged monoclonal antibodies (all Miltenyi Biotec Inc., Gladbach, Germany): Viogreen-CD3 and APC-CD8. Following washing, cells were fixed and permeabilized using CytoPerm kit (BD Bioscience), and then incubated with PE-anti-IFN- $\gamma$. Cells were analyzed with a MACSQuant analyzer flow cytometer (Milteny 
Biotec Inc.). Compensation beads were used to compensate for spectral overlap in each panel. Single color tubes were used to identify positive and negative staining by each antibody. Lymphocytes were identified by forward and side scatter characteristics and gated electronically using MACSQuantify ${ }^{\mathrm{TM}}$ software. IFN- $\gamma$ expressing cells were assessed within $\mathrm{CD}^{+}$ T-cells. Background levels of IFN- $\gamma$ expressing cells (present in control condition) were subtracted from experimental and positive controls.

\section{Statistical Analyses}

Data were screened for normality and the presence of outliers graphically and homogeneity of variance was assessed by Levene's test. Participant characteristics were compared across the three groups by ANOVA; exercise performance measures were compared across the two exercise groups by $t$-tests.

Antibody titers (geometric means) were base-2 logarithmically transformed and normality of residuals was confirmed through examination of P-P plots. Log2 titers were analyzed using random-intercepts maximum likelihood mixed models with a variance components covariance structure. Models included time (three time points), group (three groups), and the interaction of group by time. Age was included as a covariate. Group differences within each timepoint were assessed though pairwise comparisons of estimated marginal means, adjusted for multiple comparisons by the method of Sidak. Fold-change in antibody titer was calculated for each time point relative to baseline titer (6-week titer/baseline titer; 24-week titer/baseline titer) and group-effects were assessed by Kruskal-Wallis Test. Effect sizes are reported as $\varepsilon^{2}$ (Tomczak and Tomczak, 2014). Titers against each of the four virus strains were considered separately.

The change in the proportion of IFN- $\gamma$ T-cells at baseline, 6-, and 24-weeks following stimulation was assessed using Friedman's test, and Kendall's W coefficient reported as measure of effect size (Tomczak and Tomczak, 2014). The proportion of IFN- $\gamma$ T-cells at each time point were compared between the groups using the Kruskal-Wallis Test. All analyses were completed using IBM SPSS Statistics for Windows, Version 26. $p<0.05$ was accepted as significant.

\section{RESULTS}

\section{Participants and Exercise}

Twenty-nine participants (20 women) were randomized into one of three groups: No-Ex, Ex-S, and Ex-Op. Participant characteristics are shown in Table 1. Groups did not differ in these characteristics.

Ex-S and Ex-Op completed a total of 50 repetitions of the lateral raise and bicep curl exercises. In both groups, the weight was lowered from the $80 \%$ of $1 \mathrm{RM}$ used at the start of the intervention as participants fatigued and technique suffered. Ex-S and Ex-Op did not differ in exercise performance (Table 2).

Participants were asked to report the presence of any soreness in the vaccinated and unvaccinated arms for each of 7 days postvaccination. Eight of 29 participants ( 3 in No-Ex, 3 in Ex-S, and 2 in Ex-Op) reported soreness in the vaccinated arm on at least 1 day; all reports of soreness resolved within 6 days. One participant in Ex-Op also reported soreness in the non-vaccinated arm (the arm that exercised); this soreness also resolved within 6 days.

\section{Antibody Titer}

Serum collected at baseline, 6-, and 24-weeks post-vaccination was assessed by hemagglutinin inhibition assays to determine antibody titers to the four influenza strains included in the vaccine. Geometric mean antibody titers are displayed in Figure 2. No-Ex, Ex-S, and Ex-Op did not differ in antibody titer to any strain across the three time points: $\mathrm{A} / \mathrm{H} 1 \mathrm{~N} 1$, $F(4,50.56)=0.575, p=0.682 ; \mathrm{A} / \mathrm{H} 3 \mathrm{~N} 2, F(4,50.57)=0.629$, $p=0.644 ; \mathrm{B} /$ Colorado/06/2017, $F(4,51.37)=1.355, p=0.262$; and $\mathrm{B} /$ Phuket/3073/2013, $F(4,50.73)=0.338, p=0.851$. There was also no difference in antibody titer between the three visits, regardless of group assignment: A/H1N1, $F(2$, $50.53)=0.726, p=0.489 ; \mathrm{A} / \mathrm{H} 3 \mathrm{~N} 2, F(2,50.52)=1.869$, $p=0.165 ; \mathrm{B} /$ Colorado $/ 06 / 2017, F(2,51.31)=1.533, p=0.226$; and $\mathrm{B} /$ Phuket/3073/2013, $F(2,50.69)=2.693, p=0.077$. Accordingly, rates of seroprotection (antibody titer $\geq 40$ ) remained low, particularly for B/Colorado/06/2017 (Table 3).

To understand if groups differed in the change in antibody titer from baseline, we compared fold-change at 6- and 24-weeks between groups (Figure 3). Overall, rates of seroconversion (foldchange $\geq 4$ ) were low (Table 3 ). No group differences in 6- or 24 -week fold change were noted for any virus strain (Table 4), and most effect sizes were small. However, a moderate effect size was noted for fold-change at 6-weeks for B/Colorado/06/2017 $\left(\varepsilon^{2}=0.169\right)$. Visual inspection of the data suggests a trend for a greater fold-change in Ex-S (Figure 3). Mean fold increases at 6-weeks in response to B/Colorado/06/2017 were 1.08 (No-Ex), 1.93 (Ex-S), and 1.06 (Ex-Op).

Despite the low rates of seroprotection, only one participant $(\mathrm{Ex}-\mathrm{S})$ reported flu-like symptoms 18 weeks postvaccination; influenza infection was not clinically confirmed in this participant.

\section{Cell Mediated Immunity}

Regardless of group assignment, vaccination did not induce an increase in influenza-specific T-cells and effect sizes remained small [No-Ex: $\mathrm{X}^{2}(2)=4.79, p=0.091, W=0.263$; Ex-S: $\mathrm{X}^{2}(2)=0.26, p=0.878, W=0.167$; Ex-Op: $\mathrm{X}^{2}(2)=2.48, p=0.289$, $W=0.111$; Figure 4]. Groups did not differ in the proportion of IFN- $\gamma+$ T-cells at any time point [baseline: $\mathrm{H}(2)=0.29, p=0.985$, $\varepsilon^{2}=0.001 ; 6$-weeks: $\mathrm{H}(2)=0.24, p=0.889, \varepsilon^{2}=0.011 ; 24$ weeks: $\left.\mathrm{H}(2)=0.31, p=0.857, \varepsilon^{2}=0.014\right]$. T-cell responses to the positive control remained constant throughout the experiment [No-Ex: $\mathrm{X}^{2}(2)=4.75, p=0.093$; Ex-S: $\mathrm{X}^{2}(2)=3.00, p=0.223$; ExOp: $\mathrm{X}^{2}(2)=2.00, p=0.368$ ]; and did not differ between groups [baseline: $\mathrm{H}(2)=1.45, p=0.484$; 6-weeks: $\mathrm{H}(2)=0.50, p=0.781$; 24-weeks: $\mathrm{H}(2)=0.30, p=0.859$; Figure 4].

\section{DISCUSSION}

We investigated the effect of a single session of eccentricfocused resistance exercise on influenza vaccine responses in 
TABLE 1 | Participant characteristics.

\begin{tabular}{|c|c|c|c|c|c|}
\hline N (Female) & All $29(20)$ & No-Ex 10 (7) & Ex-S $10(7)$ & Ex-OP 9 (5) & $p$ \\
\hline Age (years) & $73.9 \pm 5.3$ & $70.9 \pm 5.7$ & $75.8 \pm 5.0$ & $75.1 \pm 5.3$ & 0.132 \\
\hline Height (cm) & $165 \pm 6.8$ & $168 \pm 8.4$ & $165 \pm 7.0$ & $164 \pm 4.5$ & 0.525 \\
\hline Weight (kg) & $75.0 \pm 14.6$ & $75.0 \pm 14.6$ & $80.7 \pm 16.4$ & $69.7 \pm 9.9$ & 0.269 \\
\hline Body mass index $\left(\mathrm{kg} / \mathrm{m}^{2}\right)$ & $27.3 \pm 4.9$ & $26.2 \pm 4.6$ & $29.7 \pm 5.7$ & $25.8 \pm 3.7$ & 0.189 \\
\hline Grip strength dominant (kg) & $27.4 \pm 8.5$ & $29.9 \pm 10.5$ & $27.1 \pm 5.7$ & $24.9 \pm 8.7$ & 0.450 \\
\hline Grip strength non-dominant (kg) & $25.1 \pm 6.9$ & $28.9 \pm 9.6$ & $23.7 \pm 3.7$ & $22.8 \pm 5.3$ & 0.123 \\
\hline
\end{tabular}

Values are mean $\pm S D$; $p$-value is associated with F-tests comparing No-Ex, Ex-S, and Ex-OP.

older adults. Older adults frequently have insufficient responses to the influenza vaccine and so are at increased risk for influenza infection. We hypothesized that eccentric exercise performed in the same arm subsequently vaccinated would improve protective immune responses to the vaccine. Our outcomes of interest, hemagglutinin inhibition antibody titers and influenza-specific T-cells, were compared between three groups before and after (6- and 24-weeks) vaccination with the seasonal quadrivalent influenza vaccine. Contrary to our hypothesis, no differences were found between No-Ex, Ex-S, and Ex-Op across this timeframe, indicating that an acute bout of unfamiliar eccentric resistance exercise, performed just before vaccination either in the same or opposite arm, did not influence antibody responses to the vaccine in this population of older adults.

Observations that greater physical activity levels are associated with greater immune responses following influenza vaccination have provided a rationale for chronic exercise training interventions as a means to improve vaccine responses (Kohut et al., 2002; Schuler et al., 2003; Whitham and Blannin, 2003; Keylock et al., 2007; Pascoe et al., 2014). Although 5-10 month long exercise training interventions have had moderate success in enhancing anti-influenza antibody titers (Kohut et al., 2004; Yang et al., 2007; Woods et al., 2009), the time commitment required may render such interventions impractical for broad implementation. Single sessions of exercise have also been proposed as a means of enhancing immune responses to vaccination. A moderate intensity aerobic

TABLE 2 | Resistance exercise performed by Ex-S (exercise in non-dominant arm) and Ex-OP (exercise in dominant arm).

\begin{tabular}{lccc}
\hline & Ex-S & Ex-OP & $\boldsymbol{p}$ \\
\hline 1 RM lateral raise (kg) & $2.6 \pm 0.8$ & $2.9 \pm 0.7$ & 0.471 \\
1 RM bicep curl (kg) & $4.2 \pm 1.2$ & $4.2 \pm 2.1$ & 0.715 \\
Intervention lateral raise (average of & $1.9 \pm 0.7$ & $2.2 \pm 0.7$ & 0.400 \\
50 rep) (kg) & & & \\
Intervention lateral raise (average of & $72.5 \pm 11.0$ & $75.9 \pm 8.1$ & 0.796 \\
50 rep) (\%1RM) & & & \\
Intervention bicep curl (average of & $3.1 \pm 0.7$ & $3.3 \pm 1.9$ & 0.791 \\
50 rep) (kg) & & & \\
Intervention bicep curl (average of & $75.9 \pm 9.6$ & $76.5 \pm 5.6$ & 0.862 \\
50 rep) (\%1RM) & & &
\end{tabular}

Values are mean $\pm S D$; $p$-value is associated with t-tests comparing Ex-S and EX-OP. 1RM: 1 repetition maximum estimated using Brzycki Formula; intervention weight is the average weight used across the 10 sets of 5 repetitions. exercise bout performed prior to inoculation yielded higher influenza antibody titers in young women, but not young men, 4- and 20-weeks post-vaccination (Edwards et al., 2006). In a subsequent examination by the same group it was reported that eccentric resistance exercise prior to influenza vaccination also significantly increased antibody titers 6-weeks post-vaccination by approximately $1.5 \mathrm{X}$ the control condition in young women, corresponding to a medium effect size (Edwards et al., 2007). Though the current study mirrored the eccentric-focused exercise protocol in the Edwards et al. study, effect sizes reported in the current study are small in almost all cases. Key differences which may explain this discrepancy in results include the fact that the study by Edwards et al. delayed vaccination for $6 \mathrm{~h}$ after the exercise bout, whereas the current study vaccinated participants immediately $(<5 \mathrm{~min})$ post-exercise. Delaying vaccination for several hours after exercise may allow a greater immune response to the exercise bout to develop, although local and systemic immune changes are also noted immediately (Peake et al., 2005). A study aiming to optimize the timing of the bout of eccentric exercise directly compared vaccinating immediately after exercise or delaying for $6 \mathrm{~h}$, but found no difference between any groups in the intervention, including the no-exercise control (Campbell et al., 2010).

A second difference is that the study by Edwards et al. used an exercise intensity of $85 \%$ of $1 \mathrm{RM}$ for the intervention, which was maintained for all 50 repetitions. The participants in the current study instead were only able to maintain an average intensity of $\sim 70-75 \% 1 \mathrm{RM}$ across the 50 repetitions. We selected a slightly lower goal intensity at the start of the intervention ( $80 \% 1 \mathrm{RM})$ in consideration of joint health of the older, resistance-trained naive participants and lowered intensity as technique suffered. This intensity is within ranges reported to improve lean muscle mass and muscle strength in older adults following resistance training interventions, thus demonstrating that $65-85 \% 1 \mathrm{RM}$ is safe and induces physiological response in older adults (Peterson et al., 2010, 2011). An alternative strategy could have been to maintain weight but increase intra- and inter-set rest. However, it has been suggested that an adjuvant effect from exercise-induced increases in lymph flow rate are reduced with increased rest periods; this strategy therefore would need careful investigation (BohnGoldbaum et al., 2020). Self-reports of muscle soreness were low following our intervention and did not differ in frequency from the No-Ex group, suggesting both that the exercise protocol was well tolerated in this population and that our exercise protocol may not have stimulated a local inflammatory reaction. If so, 

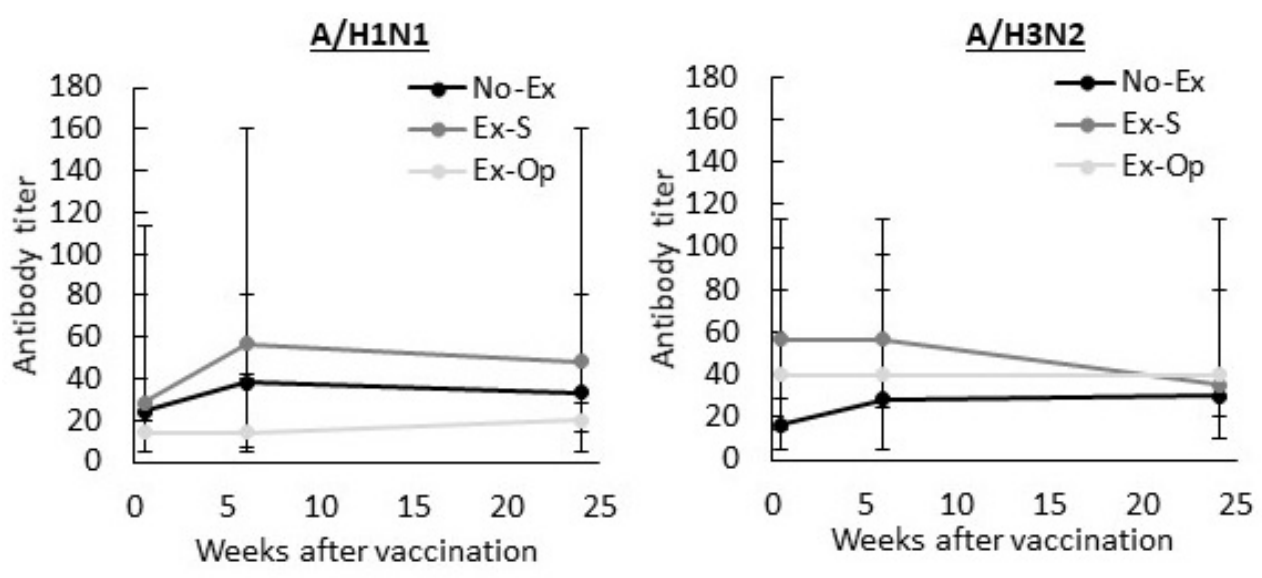

B/Colorado/06/2017
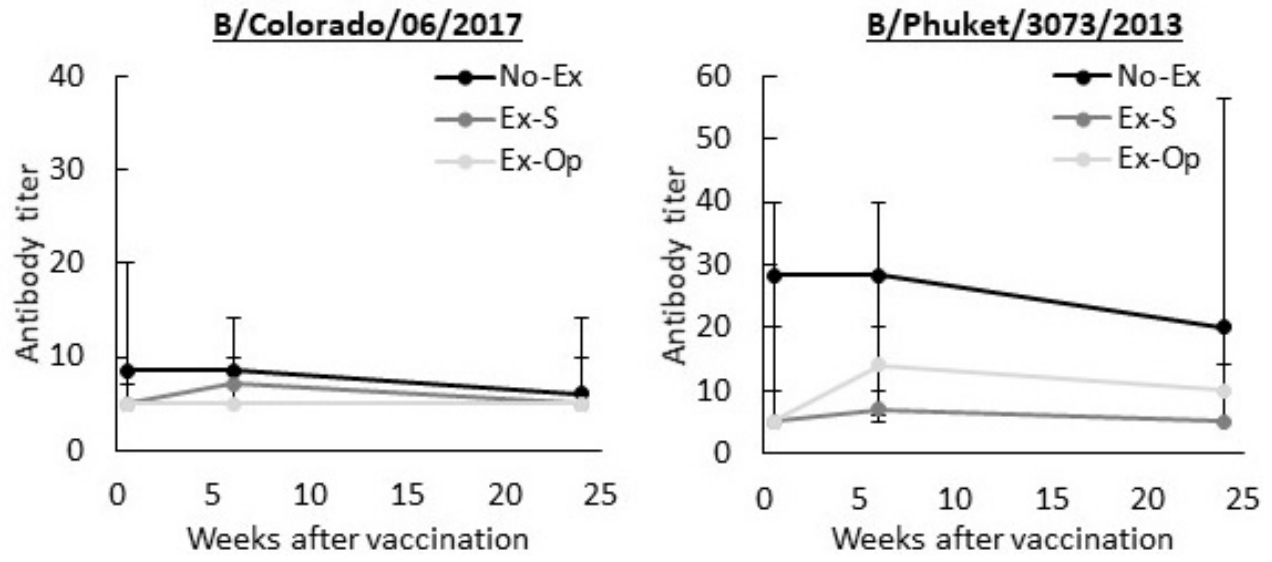

FIGURE 2 | Influenza-specific antibody titers before, 6-, and 24-weeks after influenza vaccination. Median and interquartile range of the geometric mean antibody titers for each strain shown. No-Ex, control group; Ex-S, exercise in inoculated arm; Ex-Op, exercise in arm not inoculated.

TABLE 3 | Number and proportion of participants overall and within group exhibiting seroprotection and seroconversion 6- and 24-weeks post-vaccination.

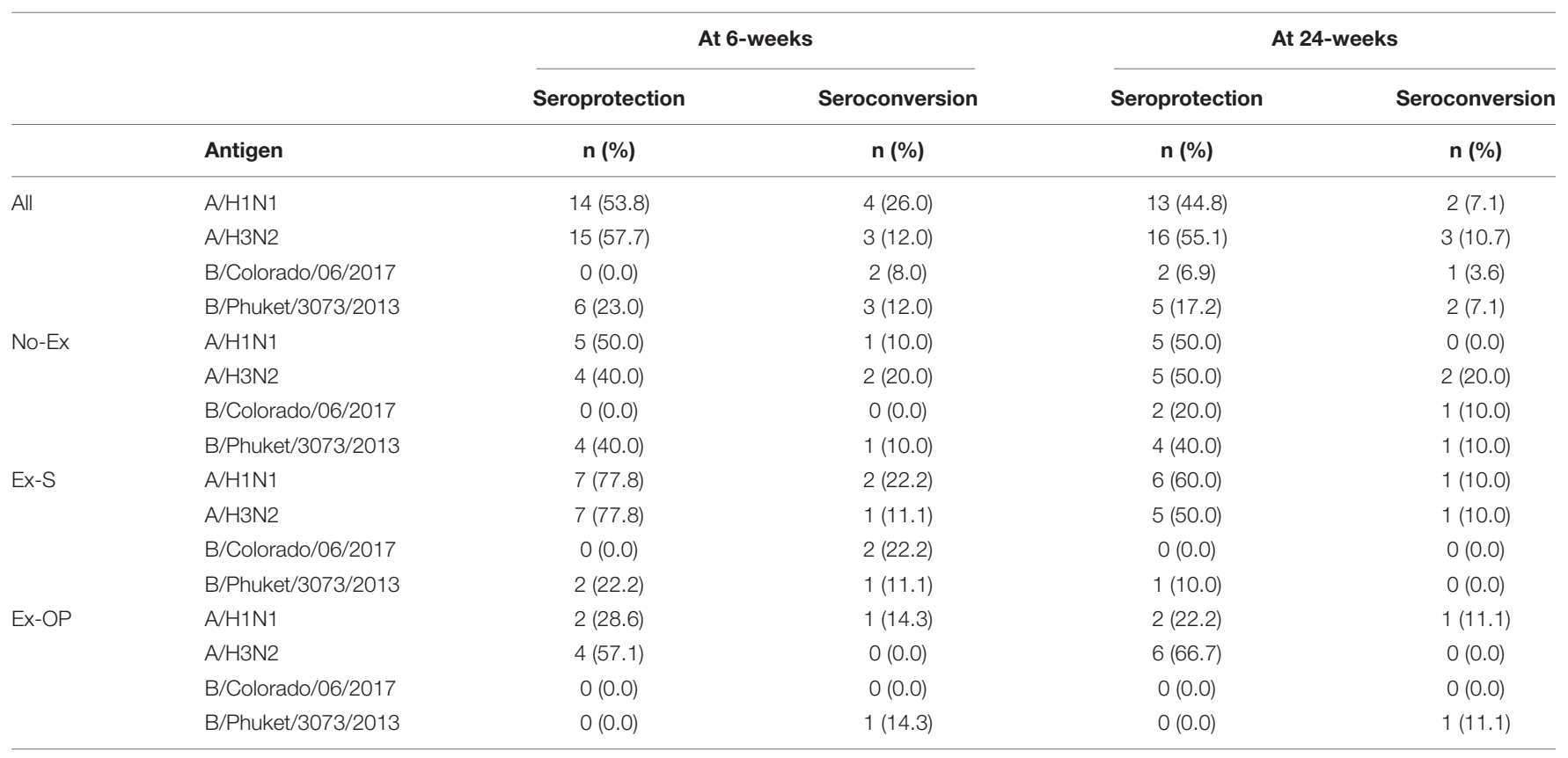

Seroprotection: antibody titer $\geq 40$; seroconversion: fold-change from pre-vaccination $\geq 4$. 


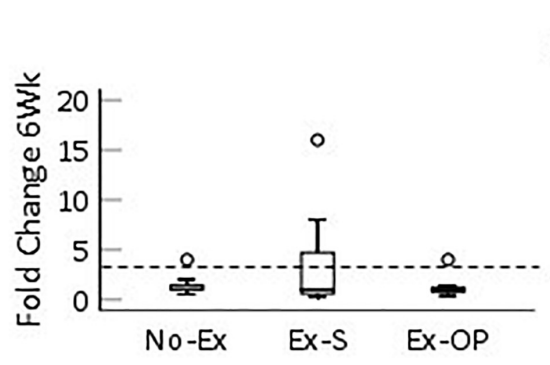

\section{A/H1N1}

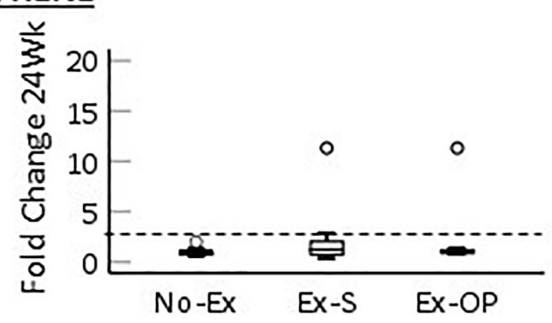

A/H3N2
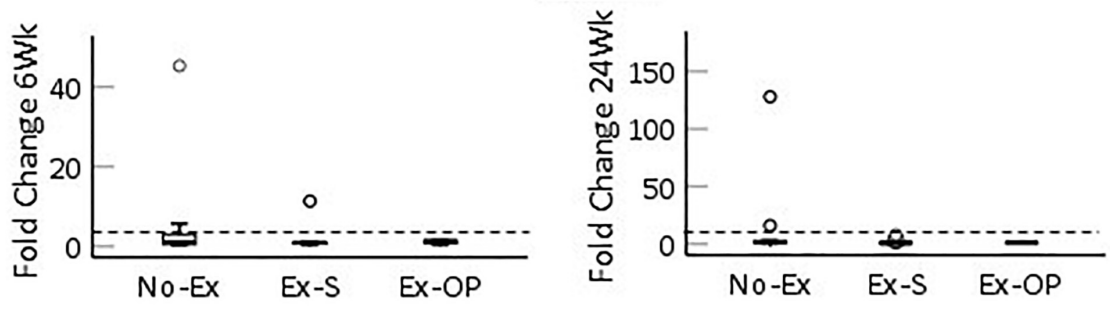

B/Colorado/06/2017
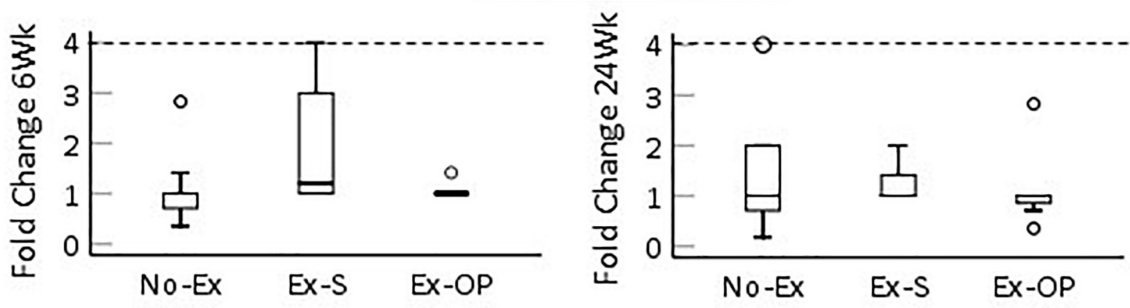

B/Phuket/3073/2013
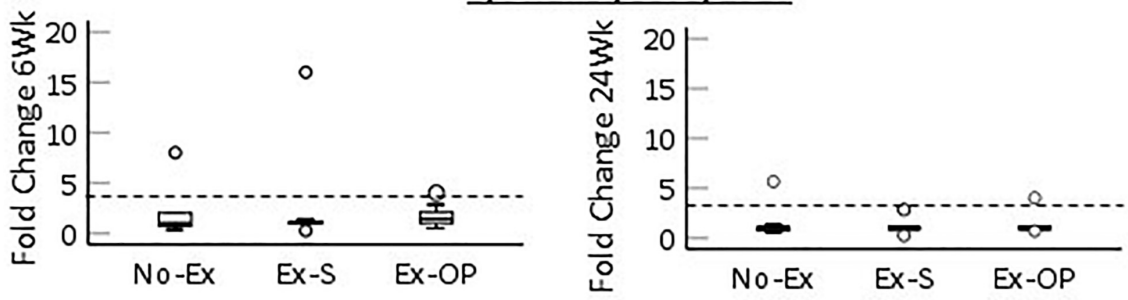

FIGURE 3 | Fold change in antibody titer from baseline 6-weeks post-vaccination (left) and 24-weeks post-vaccination (right) for each strain by group. Median (black bar), 1st and 3rd quartiles (box), confidence interval (error bar) and outliers (circles) are shown. Dashed line indicates seroconversion.

this would be counter to studies demonstrating leukocytosis and inflammation following acute resistance exercise, even at lower intensities (Nosaka and Newton, 2002; Peake et al., 2005; Szlezak et al., 2016; Chen et al., 2020; Schlagheck et al., 2020).The lack of a systemic marker of inflammation or muscle damage (e.g., creatine kinase) is a limitation to our study. Nevertheless, these differences in intensity may not have influenced antibody titers, as was demonstrated in a direct comparison of the effect of eccentric arm exercise at 60,85 , and $110 \%$ of $1 \mathrm{RM}$ on antibody responses to influenza vaccination that found no difference between exercise groups (Edwards et al., 2010).

Perhaps the largest difference between the earlier eccentricexercise investigations and the current study is the fact that our participants were older adults, rather than younger adults who typically achieved clinically protective immune responses to the vaccine even when assigned to the resting control group (Edwards et al., 2007; Campbell et al., 2010; Edwards et al., 2010). In contrast, vaccination of the older participants here largely failed to generate seroconversion, similar to other reports (Goodwin et al., 2006; Van Epps et al., 2017; Bohn-Goldbaum et al., 2020). We had hypothesized that exercise would provide a greater benefit in the current study, as others have found that exercise can enhance immune responses, especially in cases where the control response is weak (Edwards et al., 2007, 2010, 2012; Campbell et al., 2010). Although the exercise effect is not statistically significant, our data offer some support of this idea. There was a trend for an effect of group on foldchange in antibody titer at 6-weeks for B/Colorado/06/2017, 
TABLE 4 | Effect of group on fold-change in antibody titers 6- and 24-weeks post-vaccination.

\begin{tabular}{lccc}
\hline Antigen & $\boldsymbol{H}$ & $\boldsymbol{p}$ & $\boldsymbol{\varepsilon}^{\mathbf{2}}$ \\
\hline Fold-change 6-weeks & & & \\
A/H1N1 & 0.162 & 0.922 & 0.007 \\
A/H3N2 & 0.738 & 0.692 & 0.032 \\
B/Colorado/06/2017 & 4.512 & 0.105 & 0.169 \\
B/Phuket/3073/2013 & 0.734 & 0.693 & 0.032 \\
Fold-change 24-weeks & & & \\
A/H1N1 & 0.625 & 0.732 & 0.024 \\
A/H3N2 & 2.072 & 0.355 & 0.076 \\
B/Colorado/06/2017 & 1.489 & 0.475 & 0.056 \\
B/Phuket/3073/2013 & 1.232 & 0.540 & 0.047 \\
\hline
\end{tabular}

Fold-change: titer at indicated week/baseline titer, $H, p$, and $\varepsilon^{2}$ from Kruskal-Wallis tests with 2 degrees of freedom.

apparently due to the larger increase in antibody titer in ExS. The B/Colorado/06/2017 strain appeared to be the least immunogenic, as the antibody titers generated to this strain were the lowest on average of the four strains in the vaccine.

The mechanisms underlying exercise-induced improvements in vaccine responses are unknown but may act through the recruitment of immune cells, particularly antigen presenting cells, into local muscle. Eccentric exercise, particularly in those not accustomed to the exercise, results in neutrophil and macrophage infiltration into the exercised muscle which release reactive oxygen and nitrogen species and cytokines (Cannon and St Pierre, 1998; Pizza et al., 2002; Nguyen and Tidball, 2003; Peake et al., 2005). This is similar to the response to chemical adjuvants added to vaccines to enhance immune responses. Like eccentric exercise, chemical adjuvants initiate strong inflammatory reactions and immune cell recruitment at the site of the injection (Mosca et al., 2008; Calabro et al., 2011). Thus, eccentric resistance exercise targeting arm and shoulder muscle may act as an adjuvant through localized inflammation at the site of the vaccine injection. Improvements in antibodyresponses to vaccination after cardiorespiratory exercise also suggest that systemic effects of exercise may play a role in immune enhancement (Edwards et al., 2006; Pascoe et al., 2014). Exercise is well known to elicit a transient increase in leukocytes and cytokines in circulation (Walsh et al., 2011). As resistance exercise protocols used to date have primarily included exercise of the arm to be inoculated, it is impossible to separate the local and systemic exercise effects. Comparing cardiorespiratory and resistance exercise protocols is also unsatisfactory, due to difficulties in matching exercise intensity. Although we aimed to overcome these limitations in the current study by including a group matched in exercise but performed in the dominant (and therefore unvaccinated) arm (Ex-Op), the small sample and small effect sizes prevent meaningful comparison. Importantly, both local and systemic immune responses to exercise are reduced in older adults (Simpson et al., 2012). Intensitymatched cardiorespiratory exercise yields a smaller increase in leukocytosis in older adults relative to young adults (Simpson et al., 2012; Spielmann et al., 2014). Although age-related declines

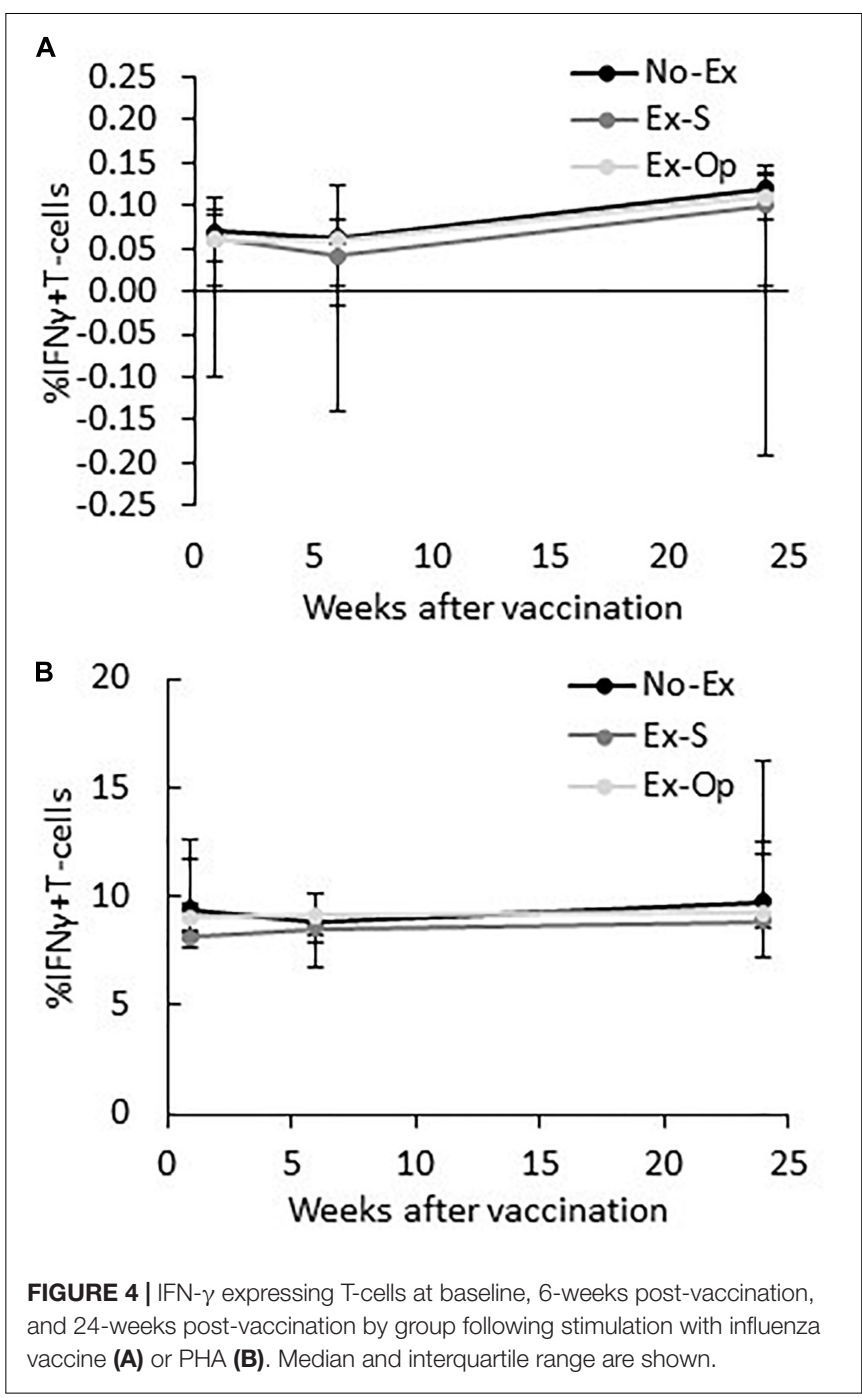

in skeletal muscle mass and strength may leave older adults more vulnerable to exercise-induced muscle damage (Manfredi et al., 1991), eccentric exercise-induced increases in circulating and muscle-infiltrating neutrophils and plasma IL-6 are reduced in older adults (Cannon et al., 1994; Toft et al., 2002; Hamada et al., 2005). Thus, it may be that the older adults in the current study did not generate as strong of an immune response to the exercise as younger participants in earlier studies using a similar exercise protocol.

A major limitation of the current study is the small number of participants relative to the effect sizes, which decreased power. The primary reason provided by interested individuals who declined participation was the convenience in receiving their seasonal influenza vaccine from their usual health care provider compared to the time required for participation. This suggests individuals are not opposed to resistance exercise per se prior to vaccination, and perhaps recruitment would be higher if it could be performed in the clinic waiting room. An additional reason for screening exclusion was for participation in the prior 6-months in arm resistance exercises. Participants were required 
to be arm resistance-training naïve to avoid heterogeneity in response due to the repeated-bout effect (Nosaka et al., 2001; Peake et al., 2005) but this did remove a segment of the older adult population that may be most receptive to performing arm resistance exercise prior to vaccination. Further studies are needed to understand if resistance-trained individuals respond differently from resistance-naïve individuals in the effects on vaccination. Additional limitations include the fact that we did not solicit information regarding vaccine reactions other than injection site soreness. A bout of resistance exercise prior to influenza vaccination in older adults has recently been found to decrease vaccine reactions such as fever (Bohn-Goldbaum et al., 2020). Thus, expanding inquiry into vaccine side effects will be important for future research.

\section{CONCLUSION}

In conclusion, we report that an acute bout of unaccustomed eccentric exercise performed immediately before vaccination with the 2018-2019 quadrivalent influenza vaccine did not yield differences in geometric mean titer, fold-increase, seroconversion, or seroprotection in older men and women, relative to a resting, standard-of-care control. Although the participants were naïve to the exercise, it was well-tolerated. The current study is limited by the small number of participants. Thus, we view these results as providing guidance for future studies targeting the use of exercise as a means of enhancing immune responses to influenza vaccine in older adults.

\section{REFERENCES}

American College of Sports Medicine, Whaley, M. H., Brubaker, P. H., Otto, R. M., and Armstrong, L. E. (2005). ACSM's Guidelines for Exercise Testing and Prescription, 7th Edn. Philadelphia, PA: Lippincott Williams and Wilkins.

Banzhoff, A., Nacci, P., and Podda, A. (2003). A new MF59-adjuvanted influenza vaccine enhances the immune response in the elderly with chronic diseases: results from an immunogenicity meta-analysis. Gerontology 49, 177-184. doi: $10.1159 / 000069172$

Beyer, W. E. P., Palache, A. M., Lüchters, G., Nauta, J., and Osterhaus, A. D. M. E. (2004). Seroprotection rate, mean fold increase, seroconversion rate: which parameter adequately expresses seroresponse to influenza vaccination? Virus Res. 103, 125-132. doi: 10.1016/j.virusres.2004.02.024

Bohn-Goldbaum, E., Pascoe, A., Singh, M. F., Singh, N., Kok, J., Dwyer, D. E., et al. (2020). Acute exercise decreases vaccine reactions following influenza vaccination among older adults. Brain Behav. Immun. Health 1:100009. doi: 10.1016/j.bbih.2019.100009

Brzycki, M. (1993). Strength testing-predicting a one-rep max from reps-to-fatigue. J. Phys. Educ. Recreat. Danc. 64, 88-90. doi: 10.1080/07303084.1993.10606684

Calabro, S., Tortoli, M., Baudner, B. C., Pacitto, A., Cortese, M., O’Hagan, D. T., et al. (2011). Vaccine adjuvants alum and MF59 induce rapid recruitment of neutrophils and monocytes that participate in antigen transport to draining lymph nodes. Vaccine 29, 1812-1823. doi: 10.1016/j.vaccine.2010.12.090

Campbell, J. P., Edwards, K. M., Ring, C., Drayson, M. T., Bosch, J. A., Inskip, A., et al. (2010). The effects of vaccine timing on the efficacy of an acute eccentric exercise intervention on the immune response to an influenza vaccine in young adults. Brain Behav. Immun. 24, 236-242. doi: 10.1016/j.bbi.2009.10.001

Cannon, J. G., Fiatarone, M. A., Fielding, R. A., and Evans, W. J. (1994). Aging and stress-induced changes in complement activation and neutrophil mobilization. J. Appl. Physiol. 76, 2616-2620. doi: 10.1152/jappl.1994.76.6.2616

Cannon, J. G., and St Pierre, B. A. (1998). Cytokines in exertion-induced skeletal muscle injury. Mol. Cell Biochem. 179, 159-167.

\section{DATA AVAILABILITY STATEMENT}

The raw data supporting the conclusions of this article will be made available by the authors, without undue reservation.

\section{ETHICS STATEMENT}

The studies involving human participants were reviewed and approved by the Institutional Review Board at the University of Houston. The patients/participants provided their written informed consent to participate in this study.

\section{AUTHOR CONTRIBUTIONS}

EL, RS, and MM designed the study. EL, ME, and MM supervised the study. EL, ME, and ML performed the data analyses. All authors contributed to manuscript writing and approve of the final version to be published.

\section{FUNDING}

This research was conducted with the support of the National Institute on Aging (Grant R03AG052778 to EL, Principal Investigator; RS, MM, and ML, Co-Investigators).

Castilla, J., Martínez-Baz, I., Martínez-Artola, V., Reina, G., Pozo, F., García Cenoz, M., et al. (2013). Decline in influenza vaccine effectiveness with time after vaccination, Navarre, Spain, season 2011/12. Euro Surveill. Bull. Eur. Mal. Transm. Eur. Commun. Dis. Bull. 18:20388.

Chen, T. C., Huang, G.-L., Hsieh, C.-C., Tseng, K.-W., Tseng, W.-C., Chou, T.-Y., et al. (2020). Comparison among three different intensities of eccentric contractions of the elbow flexors resulting in the same strength loss at one day post-exercise for changes in indirect muscle damage markers. Eur. J. Appl. Physiol. 120, 267-279. doi: 10.1007/s00421-019-042 $72-\mathrm{w}$

Cohen, S., Kamarck, T., and Mermelstein, R. (1983). A global measure of perceived stress. J. Health Soc. Behav. 24, 385-396. doi: 10.2307/2136404

Della Cioppa, G., Nicolay, U., Lindert, K., Leroux-Roels, G., Clement, F., Castellino, F., et al. (2014). A dose-ranging study in older adults to compare the safety and immunogenicity profiles of MF59 ${ }^{\circledR}$-adjuvanted and non-adjuvanted seasonal influenza vaccines following intradermal and intramuscular administration. Hum. Vaccin. Immunother. 10, 1701-1710. doi: 10.4161/hv.28 618

Edwards, K. M., Burns, V. E., Allen, L. M., McPhee, J. S., Bosch, J. A., Carroll, D., et al. (2007). Eccentric exercise as an adjuvant to influenza vaccination in humans. Brain Behav. Immun. 21, 209-217. doi: 10.1016/j.bbi.2006.04. 158

Edwards, K. M., Burns, V. E., Reynolds, T., Carroll, D., Drayson, M., and Ring, C. (2006). Acute stress exposure prior to influenza vaccination enhances antibody response in women. Brain Behav. Immun. 20, 159-168. doi: 10.1016/j.bbi.2005. 07.001

Edwards, K. M., Campbell, J. P., Ring, C., Drayson, M. T., Bosch, J. A., Downes, C., et al. (2010). Exercise intensity does not influence the efficacy of eccentric exercise as a behavioural adjuvant to vaccination. Brain Behav. Immun. 24, 623-630. doi: 10.1016/j.bbi.2010.01.009

Edwards, K. M., Pung, M. A., Tomfohr, L. M., Ziegler, M. G., Campbell, J. P., Drayson, M. T., et al. (2012). Acute exercise enhancement of pneumococcal 
vaccination response: a randomised controlled trial of weaker and stronger immune response. Vaccine 30, 6389-6395. doi: 10.1016/j.vaccine.2012.08.022

Ennis, F. A., Rook, A. H., Qi, Y. H., Schild, G. C., Riley, D., Pratt, R., et al (1981). HLA restricted virus-specific cytotoxic T-lymphocyte responses to live and inactivated influenza vaccines. Lancet (London, England) 2, 887-891. doi: 10.1016/s0140-6736(81)91389-1

Fagiolo, U., Amadori, A., Cozzi, E., Bendo, R., Lama, M., Douglas, A., et al. (1993). Humoral and cellular immune response to influenza virus vaccination in aged humans. Aging (Milano) 5, 451-458. doi: 10.1007/bf03324202

Fried, L. P., Tangen, C. M., Walston, J., Newman, A. B., Hirsch, C., Gottdiener, J., et al. (2001). Frailty in older adults: evidence for a phenotype. J. Gerontol. A Biol. Sci. Med. Sci. 56, M146-M156.

Goldberg, D. (1978). Manual of the General Health Questionnaire. Windsor: Nfer Nelson.

Goodwin, K., Viboud, C., and Simonsen, L. (2006). Antibody response to influenza vaccination in the elderly: a quantitative review. Vaccine 24, 1159-1169. doi: 10.1016/j.vaccine.2005.08.105

Hainz, U., Jenewein, B., Asch, E., Pfeiffer, K.-P., Berger, P., and GrubeckLoebenstein, B. (2005). Insufficient protection for healthy elderly adults by tetanus and TBE vaccines. Vaccine 23, 3232-3235. doi: 10.1016/j.vaccine.2005. 01.085

Hamada, K., Vannier, E., Sacheck, J. M., Witsell, A. L., and Roubenoff, R. (2005). Senescence of human skeletal muscle impairs the local inflammatory cytokine response to acute eccentric exercise. FASEB J. Off. Publ. Fed. Am. Soc. Exp. Biol. $19,264-266$.

Haynes, L., and Swain, S. L. (2006). Why aging T cells fail: implications for vaccination. Immunity 24, 663-666. doi: 10.1016/j.immuni.2006.06.003

Hays, R. D., Sherbourne, C. D., and Mazel, R. M. (1993). The RAND 36-item health survey 1.0. Health Econ. 2, 217-227.

Hoare, Z. S. J., Whitaker, C. J., and Whitaker, R. (2013). Introduction to a generalized method for adaptive randomization in trials. Trials 14:19. doi: 10.1186/1745-6215-14-19

Keitel, W. A., Atmar, R. L., Cate, T. R., Petersen, N. J., Greenberg, S. B., Ruben, F., et al. (2006). Safety of high doses of influenza vaccine and effect on antibody responses in elderly persons. Arch. Intern. Med. 166, 1121-1127. doi: 10.1001/ archinte.166.10.1121

Keylock, K. T., Lowder, T., Leifheit, K. A., Cook, M., Mariani, R. A., Ross, K., et al. (2007). Higher antibody, but not cell-mediated, responses to vaccination in high physically fit elderly. J Appl Physiol. 102, 1090-1098. doi: 10.1152/japplphysiol. 00790.2006

Kohut, M. L., Arntson, B. A., Lee, W., Rozeboom, K., Yoon, K.-J., Cunnick, J. E., et al. (2004). Moderate exercise improves antibody response to influenza immunization in older adults. Vaccine 22, 2298-2306. doi: 10.1016/j.vaccine. 2003.11.023

Kohut, M. L., Cooper, M. M., Nickolaus, M. S., Russell, D. R., and Cunnick, J. E. (2002). Exercise and psychosocial factors modulate immunity to influenza vaccine in elderly individuals. J. Gerontol. A Biol. Sci. Med. Sci. 57, M557-M562.

Leroux-Roels, I., Borkowski, A., Vanwolleghem, T., Dramé, M., Clement, F., Hons, E., et al. (2007). Antigen sparing and cross-reactive immunity with an adjuvanted $\mathrm{rH} 5 \mathrm{~N} 1$ prototype pandemic influenza vaccine: a randomised controlled trial. Lancet (London, England) 370, 580-589. doi: 10.1016/s01406736(07)61297-5

Manfredi, T. G., Fielding, R. A., O’Reilly, K. P., Meredith, C. N., Lee, H. Y., and Evans, W. J. (1991). Plasma creatine kinase activity and exercise-induced muscle damage in older men. Med. Sci. Sports Exerc. 23, 1028-1034.

Mosca, F., Tritto, E., Muzzi, A., Monaci, E., Bagnoli, F., Iavarone, C., et al. (2008). Molecular and cellular signatures of human vaccine adjuvants. Proc. Natl. Acad. Sci. U.S.A. 105, 10501-10506. doi: 10.1073/pnas.0804699105

Nguyen, H. X., and Tidball, J. G. (2003). Interactions between neutrophils and macrophages promote macrophage killing of rat muscle cells in vitro. J. Physiol. 547(Pt 1), 125-132. doi: 10.1113/jphysiol.2002.031450

Nosaka, K., and Newton, M. (2002). Difference in the magnitude of muscle damage between maximal and submaximal eccentric loading. J strength Cond Res. 16, 202-208. doi: 10.1519/1533-4287(2002)016<0202:ditmom >2.0.co;2

Nosaka, K., Sakamoto, K., Newton, M., and Sacco, P. (2001). How long does the protective effect on eccentric exercise-induced muscle damage last? Med. Sci. Sports Exerc. 33, 1490-1495. doi: 10.1097/00005768-200109000-00011
Pascoe, A. R., Fiatarone Singh, M. A., and Edwards, K. M. (2014). The effects of exercise on vaccination responses: a review of chronic and acute exercise interventions in humans. Brain Behav. Immun. 39, 33-41. doi: 10.1016/j.bbi. 2013.10.003

Peake, J., Nosaka, K., and Suzuki, K. (2005). Characterization of inflammatory responses to eccentric exercise in humans. Exerc. Immunol. Rev. 11, 64-85.

Peterson, M. D., Rhea, M. R., Sen, A., and Gordon, P. M. (2010). Resistance exercise for muscular strength in older adults: a meta-analysis. Ageing Res. Rev. 9, 226-237. doi: 10.1016/j.arr.2010.03.004

Peterson, M. D., Sen, A., and Gordon, P. M. (2011). Influence of resistance exercise on lean body mass in aging adults: a meta-analysis. Med. Sci. Sports Exerc. 43, 249-258. doi: 10.1249/mss.0b013e3181eb6265

Pizza, F. X., Koh, T. J., McGregor, S. J., and Brooks, S. V. (2002). Muscle inflammatory cells after passive stretches, isometric contractions, and lengthening contractions. J. Appl. Physiol. 92, 1873-1878. doi: 10.1152/ japplphysiol.01055.2001

Proske, U., and Morgan, D. L. (2001). Muscle damage from eccentric exercise: mechanism, mechanical signs, adaptation and clinical applications. J. Physiol. 537(Pt 2), 333-345. doi: 10.1111/j.1469-7793.2001.00333.x

Rastogi, S., Gross, P. A., Bonelli, J., Dran, S., Levandowski, R. A., Russo, C., et al. (1995). Time to peak serum antibody response to influenza vaccine. Clin. Diagn Lab Immunol. 2, 120-121. doi: 10.1128/cdli.2.1.120-121.1995

Schlagheck, M. L., Walzik, D., Joisten, N., Koliamitra, C., Hardt, L., Metcalfe, A. J., et al. (2020). Cellular immune response to acute exercise: Comparison of endurance and resistance exercise. Eur. J. Haematol. 105, 75-84. doi: 10.1111/ ejh. 13412

Schuler, P. B., Leblanc, P. A., and Marzilli, T. S. (2003). Effect of physical activity on the production of specific antibody in response to the 1998-99 influenza virus vaccine in older adults. J. Sports Med. Phys. Fitness 43:404.

Shurin, M. R., Shurin, G. V., and Chatta, G. S. (2007). Aging and the dendritic cell system: implications for cancer. Crit. Rev. Oncol. Hematol. 64, 90-105. doi: 10.1016/j.critrevonc.2007.03.002

Siegrist, C.-A., and Aspinall, R. (2009). B-cell responses to vaccination at the extremes of age. Nat. Rev. Immunol. 9, 185-194. doi: 10.1038/nri2508

Simpson, R. J., Lowder, T. W., Spielmann, G., Bigley, A. B., LaVoy, E. C., and Kunz, H. (2012). Exercise and the aging immune system. Ageing Res. Rev. 11, 404-420.

Song, J. Y., Cheong, H. J., Hwang, I. S., Choi, W. S., Jo, Y. M., Park, D. W., et al. (2010). Long-term immunogenicity of influenza vaccine among the elderly: Risk factors for poor immune response and persistence. Vaccine. 28, 3929-3935. doi: 10.1016/j.vaccine.2010.03.067

Spielmann, G., Bigley, A. B., Lavoy, E. C., and Simpson, R. J. (2014). Aging immunity and the impact of physical exercise. Immunol. Aging

Sridhar, S., Begom, S., Bermingham, A., Hoschler, K., Adamson, W., Carman, W., et al. (2013). Cellular immune correlates of protection against symptomatic pandemic influenza. Nat. Med. 19, 1305-1312. doi: 10.1038/nm.3350

Szlezak, A. M., Szlezak, S. L., Keane, J., Tajouri, L., and Minahan, C. (2016). Establishing a dose-response relationship between acute resistance-exercise and the immune system: Protocol for a systematic review. Immunol. Lett. 180, 54-65. doi: 10.1016/j.imlet.2016.10.010

Thompson, W. W., Shay, D. K., Weintraub, E., Brammer, L., Bridges, C. B., Cox, N. J., et al. (2004). Influenza-associated hospitalizations in the United States. JAMA 292, 1333-1340. doi: 10.1001/jama.292.11.1333

Thompson, W. W., Shay, D. K., Weintraub, E., Brammer, L., Cox, N., Anderson, L. J., et al. (2003). Mortality associated with influenza and respiratory syncytial virus in the United States. JAMA 289, 179-186. doi: 10.1001/jama.289.2.179

Thompson, W. W., Weintraub, E., Dhankhar, P., Cheng, P.-Y., Brammer, L., Meltzer, M. I., et al. (2009). Estimates of US influenza-associated deaths made using four different methods. Influenza Other Res. Viruses 3, 37-49. doi: 10. 1111/j.1750-2659.2009.00073.x

Toft, A. D., Jensen, L. B., Bruunsgaard, H., Ibfelt, T., Halkjaer-Kristensen, J., Febbraio, M., et al. (2002). Cytokine response to eccentric exercise in young and elderly humans. Am. J. Physiol. Cell Physiol. 283, C289-C295.

Tomczak, M., and Tomczak, E. (2014). The need to report effect size estimates revisited. An overview of some recommended measures of effect size. Trends Sport Sci. 1, 19-25.

Van Epps, P., Tumpey, T., Pearce, M. B., Golding, H., Higgins, P., Hornick, T., et al. (2017). Preexisting immunity, not frailty phenotype, predicts influenza 
postvaccination titers among older veterans. Clin. Vaccine Immunol. 24:e4 98-16.

Walsh, N. P., Gleeson, M., Shephard, R. J., Gleeson, M., Woods, J. A., Bishop, N. C., et al. (2011). Position statement. Part one: Immune function and exercise. Exerc. Immunol. Rev. 17, 6-63.

Whitham, M., and Blannin, A. K. (2003). The effect of exercise training on the kinetics of the antibody response to influenza vaccination. J. Sports Sci. 21, 991-1000. doi: 10.1080/0264041031000140464

Woods, J. A., Keylock, K. T., Lowder, T., Vieira, V. J., Zelkovich, W., Dumich, S., et al. (2009). Cardiovascular exercise training extends influenza vaccine seroprotection in sedentary older adults: the immune function intervention trial. J. Am. Geriatr. Soc. 57, 2183-2191. doi: 10.1111/j.1532-5415.2009.025 63. $\mathrm{x}$

Yang, Y., Verkuilen, J., Rosengren, K. S., Mariani, R. A., Reed, M., Grubisich, S. A., et al. (2007). Effects of a Taiji and Qigong intervention on the antibody response to influenza vaccine in older adults. Am. J. Chin. Med. 35, 597-607. doi: 10.1142/s0192415x07005090
Conflict of Interest: The authors declare that the research was conducted in the absence of any commercial or financial relationships that could be construed as a potential conflict of interest.

Publisher's Note: All claims expressed in this article are solely those of the authors and do not necessarily represent those of their affiliated organizations, or those of the publisher, the editors and the reviewers. Any product that may be evaluated in this article, or claim that may be made by its manufacturer, is not guaranteed or endorsed by the publisher.

Copyright (C) 2021 Elzayat, Markofski, Simpson, Laughlin and LaVoy. This is an open-access article distributed under the terms of the Creative Commons Attribution License (CC BY). The use, distribution or reproduction in other forums is permitted, provided the original author(s) and the copyright owner(s) are credited and that the original publication in this journal is cited, in accordance with accepted academic practice. No use, distribution or reproduction is permitted which does not comply with these terms. 